\title{
Des œufs mués : entendre omelette
}

\author{
Gabriel Bergounioux \\ Centre Orléanais de Recherche en Acoustique et Linguistique (CORAL), Université d'Orléans \\ gabriel.bergounioux@univ-orleans.fr
}

\section{Introduction}

Un problème récurrent en linguistique concerne la nature de la relation à établir entre les représentations conventionnelles de la phonologie et les réalisations dans le discours, entre la langue et la parole en termes saussuriens (e.g. Gadet 1989). Généralement, on part de la forme de la cible phonologique qu'on apparie à ses occurrences dans un corpus. La distance entre la notation formalisée (type) et les occurrences (tokens) devient alors susceptible d'une explication qui pourra être, selon le cas :

- phonologique (par exemple concernant la résistance relative des segments dans la chaîne, cf. Ségéral \& Scheer, 2001),

- phonétique (e.g. les assimilations dans une conception articulatoire),

- morphologique (interactions entre dérivation et contraintes phonotactiques),

- $\quad$ sociologique (cf. le traitement de la liaison par P. Encrevé (1988)),

- $\quad$ voire psychanalytique (lapsus, calembour).

A partir d'un exemple concret, celui du mot omelette relevé dans le corpus ESLO qu'on présentera pour commencer $(\S 1)$, on se propose de reprendre l'analyse des items en contrastant réalisations et notation phonologique $(\S 2)$, puis, en fonction d'hypothèses de travail réinterprétant la variation sociolinguistique en termes phonologiques $(\S 3)$, on procèdera à l'inventaire des réalisations $(\S 4)$ avant de proposer une interprétation sociologique des données $(§ 5)$.

\section{Présentation d'ESL01 : la question de l'omelette}

\subsection{L’Enquête Socio-Linguistique à Orléans}

A la fin des années 60, une équipe d'universitaires anglais décide de constituer, à des fins pédagogiques, une série d'enregistrements du français en mettant l'accent sur la variété des usages qu'ils saisissent en jouant sur différents paramètres :

- protocole d'enregistrement (avec micro caché par exemple),

- situation d'entretien (interviews, débat, dîner de famille, appel téléphonique...),

- familiarité relative avec les témoins (dans les reprises de contact),

- indicateurs sociologiques : âge, sexe, catégorie socio-professionnelle.

La différenciation géographique, sans être écartée, est seulement prise en compte de façon oblique. Au total, la collecte s'étend sur quatre ans et accumule 350 heures archivées sur bandes magnétiques, la moitié du temps environ correspondant à 148 entretiens menés sur questionnaires auprès de locuteurs identifiables.

Cette enquête, dite ESLO (Enquête Socio-Linguistique à Orléans, désormais ESLO1), met à disposition un document unique sur l'état du français ordinaire il y a quarante ans, et témoigne aussi des attentes en matière de didactique et de sociolinguistique à l'époque. Il y a, dans les ambitions du projet, une générosité militante (faciliter l'apprentissage des langues vivantes, briser les modèles académiques fondés sur l'usage littéraire, développer les échanges entre les peuples) et une justification scientifique : la 
sociolinguistique de B. Bernstein (cf. Bernstein 1975) dont les hypothèses seront progressivement infléchies par la fréquentation du Centre de Sociologie Européenne de P. Bourdieu.

Dans le questionnaire d'entretien, qui se décompose en trois parties :

- une déclinaison des identifiants hors micro (questionnaire fermé),

- une série de questions obligées dites «tronc commun » découpées en séquences et

- des questions facultatives, dites «branches », sollicitées en cas d'intérêt supputé de l'interviewé pour le thème,

une branche, dite «langue et culture», dévide huit questions dont la première commence par: «Un étranger veut venir en France pour apprendre le français. Dans quelle région est-ce qu'il doit aller d'après vous ? » et dont la dernière est ainsi formulée : «Comment est-ce qu'on fait une omelette ? Pourriez-vous m'expliquer comment on fait ? / Pouvez-vous me donner la recette de l'omelette? ». C'est la seule question qui se trouve souvent précédée d'un commentaire de l'enquêteur annonçant qu'il ne s'agit pas forcément d'une interrogation « sérieuse ».

Tous les témoins n'ont pas été testés sur leurs qualités culinaires mais 96 ont été sollicités et cet échantillon a été exploité afin de recenser des items d'omelette. On n'a compté, pour les occurrences, ni celles des interviewers, ni celles de personnes extérieures qui assistaient à l'échange.

Tableau 1 Répartition des occurrences d'omelettes

Nombre de personnes interviewées 148

Nombre d'entretiens incluant la question « omelette » 96

Nombre de témoins utilisant le mot omelette

Locuteurs utilisant omelette 0 fois

$\begin{array}{lr}1 \text { fois } & 19 \\ 2 \text { fois } & 20 \\ 3 \text { fois } & 16 \\ 4 \text { à } 6 \text { fois } & 15 \\ 7 \text { à } 13 \text { fois } & 9\end{array}$

\subsection{La composition sociale de l'échantillon}

L'intérêt premier d'ESLO1 est la connaissance des caractérisations sociologiques. Ainsi, pour les 79 témoins, on peut distribuer le panel en cinq groupes:

Tableau 2 Composition sociale de l'échantillon ( $\mathrm{n}=79$ personnes)

Groupe A $=11$ personnes (+ 4 n'ont pas employé omelette)

Niveau d'études : Bac et plus

Revenus supérieurs (par rapport à l'échantillon)

Groupe $\mathrm{E}=19$ personnes ( +3 n'ont pas employé omelette)

Niveau d'études : Brevet ou équivalent

Revenus intermédiaires 
Groupe I $=14$ personnes ( +4 n'ont pas employé omelette)

Niveau d'études : Brevet ou équivalent

Revenus supérieurs

Groupe $\mathrm{O}=27$ personnes ( +5 n'ont pas employé omelette)

Niveau d'études : Certificat d'Etudes Primaires ou équivalent

Revenus modestes

Groupe $\mathrm{U}=8$ personnes ( +1 n'a pas employé omelette)

Niveau d'études : Certificat d'Etudes Primaires ou moins

Revenus très modestes

Comme pour tout classement social, les lignes de partage sont discutables. Celles retenues mettent néanmoins en évidence une dissymétrie entre les témoins des groupes intermédiaires ( $\mathrm{E}$ et $\mathrm{O})$ d'une part, et les groupes ayant le plus faible (U) et les plus forts revenus (A et I). Dans l'intention des concepteurs, qui avaient réinterprété leur panel conçu en collaboration avec l'INSEE selon une échelle dite AM distribuée en cinq groupes, de A à E, ceux-ci devaient être équivalents numériquement. Le groupe le plus dominé est le moins bien représenté, ce qui reflète l'importance du taux de refus enregistré auprès des personnes sollicitées. Les deux groupes dominants, économiquement ou culturellement, qui ont au contraire un taux de participation supérieur à la moyenne, ne sont pas si présents : y a-t-il là l'indice d'une certaine réticence, de la part des enquêteurs, à solliciter des témoins d'un rang social plus élevé sur une activité aussi triviale (et féminine) que la cuisine?

Avant de corréler telles propriétés sociales à des réalisations, on proposera une formalisation de la cible phonologique activée par la perception pour réduire l'allomorphisme des occurrences d'omelette, tant dans la représentation mentale que dans la notation symbolique. On traitera ensuite de la transcription phonétique et de son établissement.

\section{Variations : perception, évaluation sociale et phonologie}

\subsection{La consonne initiale d'omelette}

Omelette a peu de chance d'apparaître isolé. Le mot est inséré dans un syntagme et quand la surprise de nombre d'auditeurs, à l'énoncé de la question, se résout par une demande de confirmation : «Une omelette ? », en structure averbale donc, le déterminant et le nom restent soudés, avec la montée d'un élément consonantique dans l'attaque vide. Il ne se rencontre aucune initiale vocalique et la présentation ordinaire du mot, la vedette dans le dictionnaire qu'on donne pour l'équivalent approché du stock lexical mental, n'est jamais réalisée telle quelle. Le remplissage par du matériel consonantique résulte de liaisons (en $/ \mathrm{n} /$ ou $/ \mathrm{z} /$ ), d'enchaînements (en $/ \mathrm{n} /, / \mathrm{m} /, / \mathrm{t} /, / \mathrm{tR} /$ ou $/ \mathrm{l} /$ ) ou d'élisions (en $/ \mathrm{l} / \mathrm{ou} / \mathrm{d} /$ ), soit, par ordre décroissant des formes de surface : nomelette / lomelette / zomelettes / tromelette / tomelette / domelette(s) / momelette

Ces consonnes, qui ne sont pas intégrables dans la définition phonologique du mot, sont parfaitement conservées en surface (sauf réduction de /tR/ à /t/, cf. Laks 1977), conformément au comportement des attaques en Coda-Miroir.

A six occurrences près, ces consonnes sont des coronales. Ni les labiales, ni les palato-vélaires ne sont recrutées. Il est d'autant plus remarquable que le /s/ soit aussi exclu. Ainsi se dessinerait une ligne de partage entre des consonnes, coronales, à valeur préférentiellement morphologique (déterminant, marque 
de pluriel) et des consonnes (labiales et palato-vélaires) à valeur plus intrinsèquement « sémantique ». Le degré de résistance de celles-ci devrait être supérieur en ce qu'elles codent une information de contenu.

Tableau 3 Les consonnes de remplissage à l'initiale

Récapitulatif (les chiffres entre parenthèses indiquent le nombre d'occurrences)

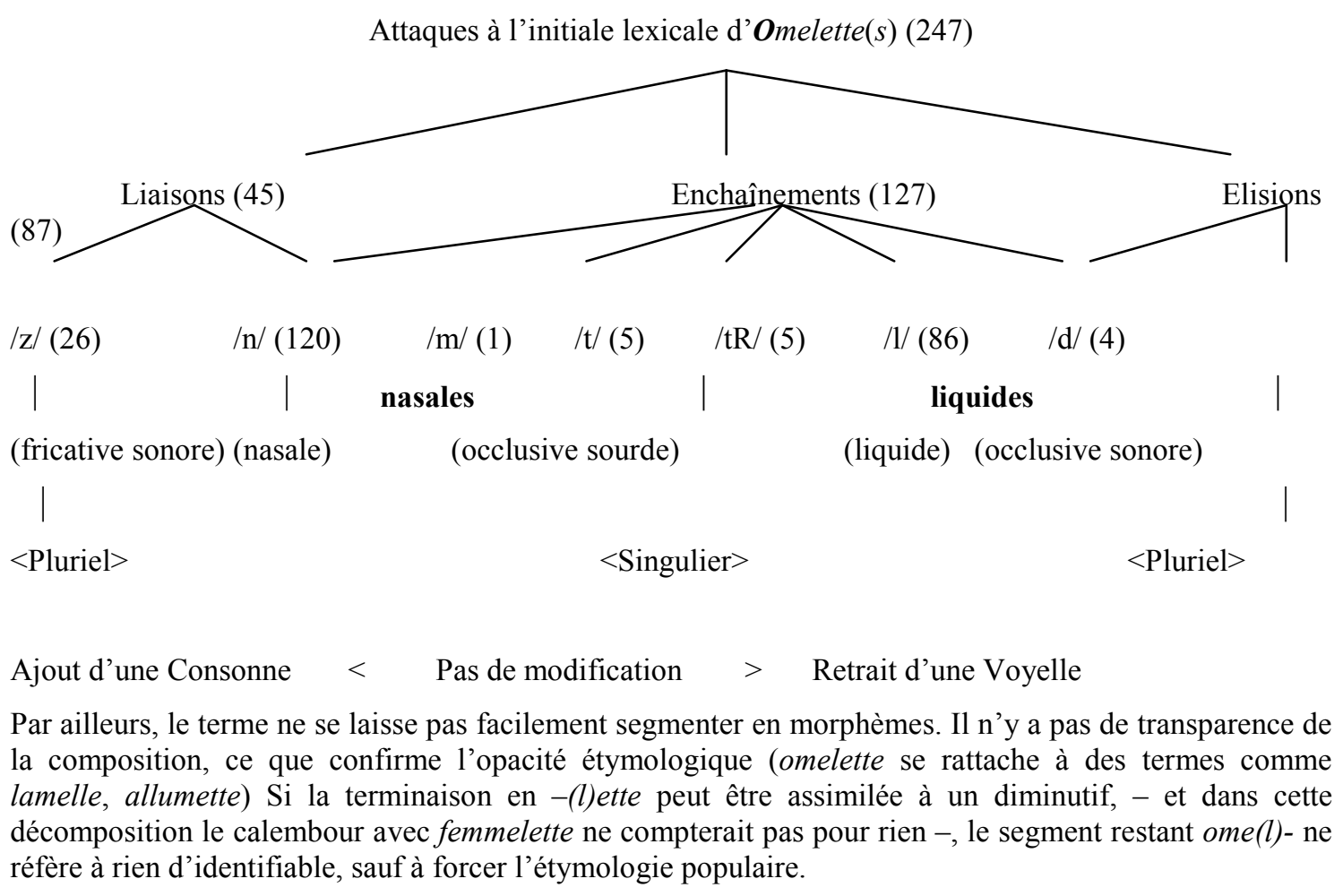

\subsection{Notation phonologique}

Dans une notation type CVCV avec squelette (Scheer, 2004), on s'accordera sur la notation suivante.

Tableau 4 Notation phonologique d'omelette

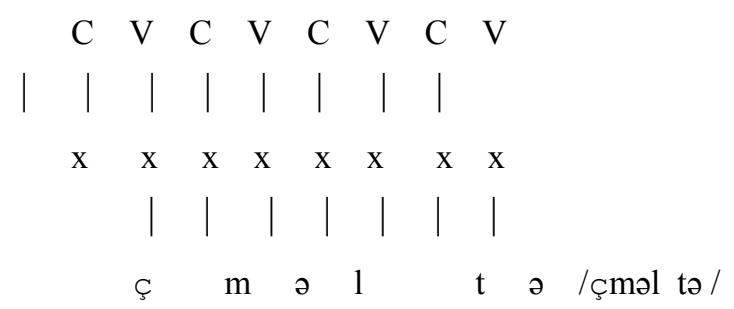

En conformité avec la phonologie du français, on retiendra les loci de variation :

- attaque initiale : remplissage par du matériel consonantique (cf. supra), 
- $\quad /$ / / : aperture conditionnée par la réalisation du schwa suivant,

- $\quad$ /a/ : vocalisation ou amuïssement du schwa central,

- $\quad$ / / : aperture conditionnée par le maintien de la consonne finale,

- $\quad / t$ / : lénition de la consonne finale,

- $\quad$ apparition d'une consonne de liaison /z/ à droite.

Aucune occurrence ne fait apparaître la marque du pluriel en liaison, les contextes propices à une réalisation étant très peu nombreux. Les quatre éléments vocaliques sont susceptibles d'une variation, neutralisation d'aperture ou effacement, et le /t/ en Coda occupant une position de lénition, seuls $/ \mathrm{m} /$ et $/ 1 /$, deux sonantes, seraient préservés, confirmant la conclusion tirée de la répartition des consonnes entre morphologie et sémantique. Si une consonne à valeur morphologique (coronales) peut être recrutée pour différencier deux racines, à l'inverse, une consonne à valeur sémantique (labiales et palato-vélaires) sera difficilement exploitable (ce qui ne veut pas dire que le cas ne se présente jamais) pour produire de la morphologie. Dans une coupe intralexicale où -ette est perçu comme un diminutif, le rattachement $\mathrm{du} / \mathrm{l} /$ à la racine ou au suffixe illustre l'ambivalence de son statut.

\subsection{Transcription phonétique}

La représentation phonologique étant admise, comment opérer pour assigner une description phonétique aux réalisations? La notation par les locuteurs eux-mêmes étant exclue et le recours aux spectrogrammes n'ayant qu'une valeur négative (elle permet d'écarter des transcriptions que démentent les images, elle ne peut décider catégoriquement de l'effet acoustique sur un auditeur), j'ai procédé à une écoute reproduite aussi souvent que nécessaire jusqu'à stabilisation d'une transcription, avant de procéder à une vérification au moyen d'une ou plusieurs réécoutes en aveugle (sans avoir sous les yeux la première transcription, donc).

Cette façon de faire a des limites qui ont souvent conduit à l'écarter. Elle présente cependant deux avantages : primo, d'impliquer celui qui s'y applique (qu'on imagine l'effet qualitativement dévastateur de plus de deux cents occurrences d'omelette présentées chacune trois fois à un panel d'auditeurs conséquent en termes d'attention et de qualité de la restitution, et ce que signifierait déjà la constitution d'un groupe de transcripteurs compétents en API). Secundo, on conserve à l'observation sa cohérence.

On rappellera apologétiquement l'exemple de l'Atlas Linguistique de la France, tout entier dressé à partir de l'unique écoute d'un mot inséré dans une liste qui en comprenait plus de mille et dans un parler dont l'auditeur (E. Edmont) ne maîtrisait pas les subtilités. Gilliéron avait même interdit à Edmont de reposer la question ou de noter une deuxième réalisation du même mot par le même locuteur. Reportés sur autant de cartes qu'il y avait d'items, c'est la seule enquête qui ait abouti en France à une carte dialectologique complète avant 1970 et les insuffisances ponctuelles, jamais rédhibitoires, ne diminuent pas la valeur incontestable qui est reconnue à l'ensemble (Le Berre et al. 2004), quoi qu'en ait pensé Rousselot.

\section{Hypothèse de travail}

\subsection{Sociolinguistique et phonologie}

L'hypothèse qui sous-tend ce travail est sociolinguistiquement phonologique, ou phonologiquement sociolinguistique. Contrastant avec PFC (Phonologie du Français Contemporain) qui met l'accent sur une variation spatiale, ou avec CLAPI, qui évalue les effets des interactions, ESLO1 privilégie une variation sociale en un même lieu et dans un même contexte d'échange, dans l'attente d'une comparaison diachronique au moyen d'ESLO2 (programme Variling de l'appel ANR «Corpus en sciences humaines $2006 »)$. 
Au lieu d'être rapportée à la distribution territoriale ou au type d'échange, la variation est assignée au moment de la perception, quand un signal miroir, généré en interne à partir d'un patron phonologique, est superposé au signal perçu en fonction :

- de paramètres contextuels probabilistes,

- des propriétés des segments (valeur potentiellement morphologique et combinabilité),

- de l'analyse acoustique effectuée.

L'opération est sociologique en ce qu'elle se situe dans une relation d'écoute phonologiquement constituée, à l'intérieur de laquelle les variations sont appréhendées comme le produit (et donc le marqueur) d'une origine dialectale, d'un niveau social et d'une relation dialogique qui se réalisent dans la structure d'une langue, dans la dimension de ce que Bourdieu appelle un habitus (Bourdieu 1979). Elle est phonologique en ce qu'elle transcende les variations, qu'on les saisisse par les propriétés du signal ou par la restitution de la perception, pour reconstituer la propension inégalement partagée de distorsion d'une cible mentale partagée par une communauté d'auditeurs jusqu'à sa fragmentation dialectale. Autrement dit, la variation aussi est, dans son principe, phonologique.

\subsection{Morpho-phonologie de la cible et des variations}

En première approche, on considèrera que la cible mentale de l'auditeur est homologue à la représentation donnée dans le tableau 4. On fait la conjecture qu'il s'agit d'un formalisme analogue à ce que produit le système d'analyse en tant qu'il fonctionne comme un procès de projection sur le signal à des fins de comparaison. Il reste cependant à enrichir la notation CVCV, qui fonde la syllabation, par des informations supplémentaires concernant la valeur des segments (cf. Scheer, 2004: 7-63, 87-93 et passim).

L’orientation privilégiée, dans la confrontation entre données phonétiques et représentation phonologique, a fait prévaloir le principe d'une dissymétrie : un schéma phonologique contraint (et potentiellement uniforme), épelé comme succession de phonèmes, s'opposerait à la dispersion des réalisations en discours. Selon cette approche, la variation serait imputable aux réalisations, aux conditions de l'énonciation, à la performance, à la parole. A partir de l'exemple d'un locus, on illustrera une autre conception : déterminer en quoi les transformations de la cible phonologique s'inscrivent comme la conséquence, empiriquement vérifiable, d'une réponse elle-même phonologiquement conditionnée, assignable à la nature des segments et à la construction morphologique des unités.

Dans cette perspective, la morphologie concerne à parts égales la préservation d'un nombre limité d'oppositions phonologiques identificatoires (Qu'est-ce qui doit être actualisé au minimum pour qu'un mot soit identifié comme tel ? Quel est le signifiant d'un morphème ? Voyelles et consonnes jouent-elles un rôle équivalent ?) et l'assignation des segments à leur capacité constructionnelle. Ainsi, un /p/ (ou un $/ \mathrm{g} /$ ) et un $/ \mathrm{t} /$ ne sont pas tenus pour équivalents, celui-ci étant souvent requis à des fins de dérivation (clou $>$ cloutier, café > cafetière...), de liaison (c'est évident), ou de flexion (il part > qu'il parte, petit > petite), ceux-là peu ou pas. La hiérarchisation de la valeur des phonèmes doit être intégrée avant d'opérer sur les chaînes.

Au nombre des opérations effectuées sur les segments, l'une des plus essentielles, en ce qu'elle révèle la dynamique interne au système, c'est-à-dire la décomposition du procès de projection avant toute détermination sociologique, concerne la neutralisation. Celle-ci remonte une opposition phonologique, actualisée par la cible, jusqu'à la corrélation immédiatement supérieure et, en réitérant l'opération, jusqu'à la distinction radicale des voyelles et des consonnes, récursivement. L'état $\mathrm{C}$ ou $\mathrm{V}$ d'une position, selon la disposition syllabique, transforme une voyelle en glide, une sonante en centre de syllabe. La neutralisation n'est pas spécifiquement centrée sur la production ; elle est présente, de la même façon, dans l'audition. 


\subsection{Un observatoire de la morphologie : amorces et rectifications}

Pour un exemple en abrégé de l'organisation morpho-phonologique d'omelette et des stratégies de réparation, on se réfèrera aux rectifications et aux amorces. Quantitativement, elles représentent moins de $3 \%$ des occurrences, soit 7 réalisations. L'hypothèse est que ces quelques formes ne sont pas sans enseignement dans la découpe de la séquence ou dans le type d'erreur qu'on peut être amené à commettre et à rectifier. Autrement dit, non seulement on ne se trompe pas n'importe comment (personne ne dit 《*èmelette » ou «*omerette » pour « omelette ») mais la rectification entreprise par le locuteur lui-même démontre quelles sont ses capacités à percevoir les limites de la variation et à rejeter une forme qu'il vient de réaliser.

Tableau 5 Rectifications et amorces

\begin{tabular}{|c|c|c|c|c|c|}
\hline & oin & Sexe & Né en & CSP & Extrait \\
\hline 1. & 045 & M & 1908 & I & «on fait une om » \\
\hline 2. & 045 & & & & « elle fait l'om » \\
\hline 3. & 076 & $\mathrm{~F}$ & 1903 & I & « la l'omelette » \\
\hline 4. & 123 & $\mathrm{~F}$ & 1936 & $\mathrm{E}$ & « le l'omelette » \\
\hline 5. & 131 & $\mathrm{~F}$ & 1900 & $\mathrm{U}$ & «l'o l'omelette »/lolçməl t/ \\
\hline 6. & 136 & $\mathrm{~F}$ & 1913 & $\mathrm{O}$ & « mon om mes œufs » \\
\hline 7. & 149 & M & 1942 & $\mathrm{O}$ & « des om des omelettes »/dezom dezçməl t/ \\
\hline
\end{tabular}

Cinq groupes: $A, E, I, O, U, A$ correspondant aux classes élevées (activité professionnelle de niveau licence), $U$ aux personnes sans qualification.

L'autocorrection du locuteur correspond à quatre phénomènes différents :

- $\quad$ interruption d'une séquence au milieu du mot au profit d'un autre énoncé (1 et 2),

- $\quad$ remplacement du nom par un autre jugé plus approprié (6),

- modification du déterminant pour aboutir à l'élision (3 et 4),

- $\quad$ reprise du /o/ initial pour restituer un timbre ouvert au lieu de fermé ( 5 et 7).

Les quatre derniers exemples circonscrivent un problème en lien avec l'organisation phonotactique, soit l'élision (remplissage de l'attaque vide), soit l'aperture du /o/ initial (en relation avec le traitement du schwa suivant, pour la détermination du statut ouvert ou fermé de la syllabe). Une autre conséquence peut s'en déduire qui concerne la segmentation interne de l'unité : sur les cinq cas où une coupe intervient, elle se produit quatre fois après le $/ \mathrm{m} /$ et une fois après le $/ \mathrm{o} /$. Encore, dans ce dernier cas, la coupe a-t-elle pour conséquence de rectifier l'aperture du /o/, elle-même conditionnée par la solidarité avec la consonne qui suit. Ainsi, s'il n'y a pas de morphologie interne immédiatement perceptible faute de transparence des composants, il y en a une sous-jacente qui suit la segmentation centrale du mot par un schwa, et que révèlent les rectifications : on découperait «om(e) - lette », comme si les caractéristiques phonologiques venaient supplée l'absence de morphologie. 


\section{Réalisations du noyau initial et de la première attaque pleine}

\subsection{Avatars du / /}

Quelles sont les réalisations phonétiques de l'initiale vocalique d'omelette? Indépendamment du remplissage de l'attaque, qui n'est pas complètement indifférent au processus, on relève sept réalisations différentes.

Tableau 6 Réalisations du noyau initial d'omelette sur 265 occurrences

- 134 occurrences de /Ç/

- 56 occurrences de $/ \propto /$

- 43 occurrences de /o/

- 14 occurrences de /a/

- 7 occurrences de /ç)/

- 6 occurrences de $/ \varnothing /$

- 5 occurrences de $/ \mathrm{m} /$

On fait l'hypothèse que la fréquence des occurrences dessine l'ordre prescrit pour les neutralisations, c'est-à-dire la composition ordonnée de chacun des segments. La forme la plus attestée /ç/ correspondrait à celle de la cible phonologique à un moment donné ( $c a$ 1970) dans les grammaires d'auditeurs. On étudiera les variations en partant d'une analyse en «valeurs» (oppositives, relatives et négatives), le signe « moins » notant qu'il ne s'agit pas de « propriétés distinctives » à valeur substantielle.

Tableau 7 Neutralisation des propriétés du/ç/ dans omelette

\begin{tabular}{|c|c|c|c|c|c|}
\hline /Ç/ & - antérieur & - fermé & - étiré & - nasal & - consonne \\
\hline /œ/ & - postérieur & - fermé & - étiré & - nasal & - consonne \\
\hline /o/ & - antérieur & - ouvert & - étiré & - nasal & - consonne \\
\hline /a/ & - postérieur & - ouvert & - labial & - nasal & - consonne \\
\hline /Ç) / & - antérieur & - fermé & - étiré & - oral & - consonne \\
\hline$|\varnothing|$ & - postérieur & - ouvert & - étiré & - nasal - & consonne \\
\hline$/ \mathrm{m} /$ & - postérieur & - ouvert & - étiré & - oral & - voyelle \\
\hline
\end{tabular}

On note que la syllabation en CVCV, où la présence du schwa est contrainte phonotactiquement (pas de séquence $/ *-\mathrm{ml}-/)$, ne se répercute pas sur le coupe syllabique puisque l'aperture du /c/ actualise un traitement qui correspond à celui d'une réalisation en syllabe fermée, que la forme de surface ait conservé ou non l'antériorité. D'autre part, alors qu'on s'attendrait à une moindre conservation de l'opposition d'aperture des voyelles moyennes, celle-ci est mieux conservée que l'opposition d'antériorité/postériorité. Est-ce une question de distribution dans la chaîne sonore ou un trait inhérent?

\subsection{L'axe des variations}

Cette série de neutralisation ordonnée hiérarchiquement par degré de fréquence, traitée avant tout considérant sociologique, montre : 
- que la moitié des réalisations, les deux tiers si on considère que dans ce contexte le degré d'aperture de la voyelle initiale n'est pas spécifié, sont conformes à la cible phonologique ;

- que le quart s'en écarte par la perte du trait/- antérieur/,

- que le restant neutralise les traits /- étiré/, /- labial/, /- oral/ et /- consonne/,

en sorte qu'on peut établir une pondération régressive des traits par leur atteinte relative dans les phénomènes de neutralisation.

Tableau 8 Les variations du/ç/

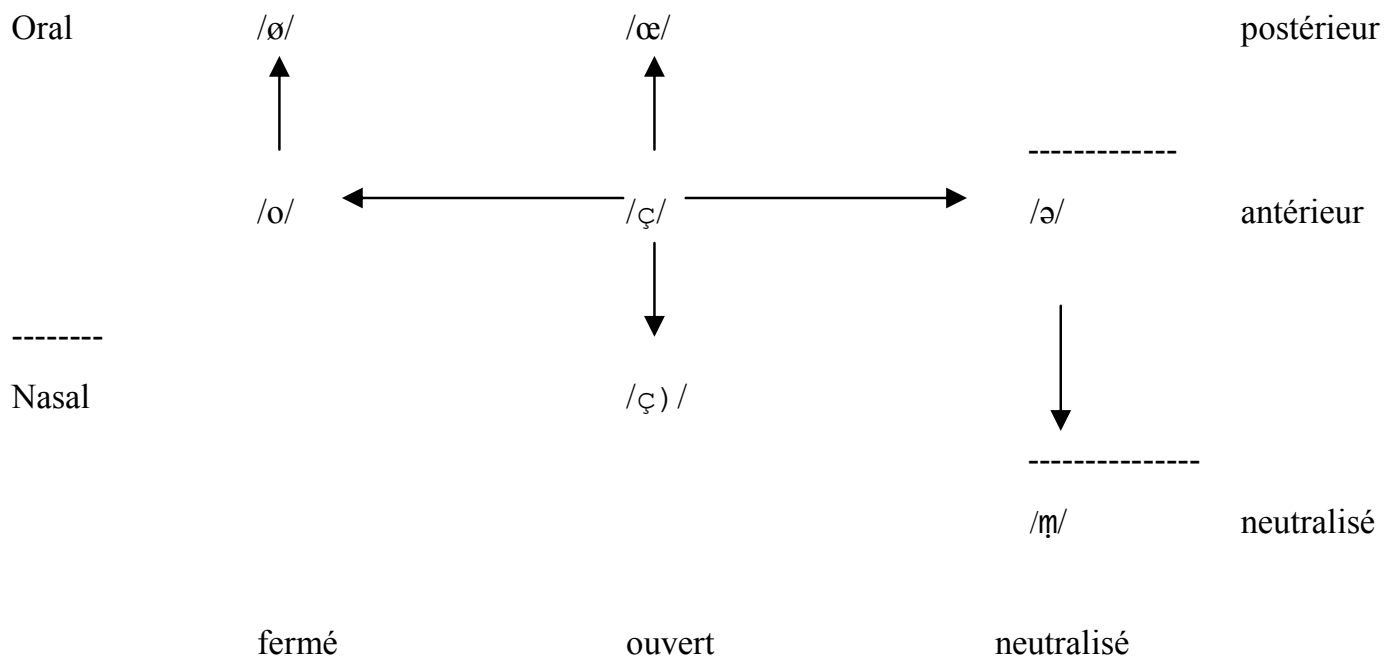

Le tableau 8 met en évidence les types de variation comme successions de transformations opérant sur les valeurs de la voyelle cible /ç/. Il s'en déduit une organisation phonologique sous-jacente qu'il est possible de corréler aux caractéristiques sociales des locuteurs.

\subsection{Nasalisation, épenthèse et vocalisation du /ə/}

Auparavant, on poursuivra l'étude sur le segment qu'ont dessiné les amorces. Jusqu'au /ə/ central, deux réalisations marginales sont attestées :/çbl $\mathrm{t} / \mathrm{ou} / \mathrm{c}$ ) bl t/ let / çmøl t/.

Tableau 9 Vocalisation du/a/

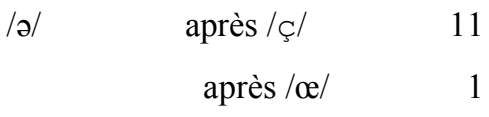

La douzaine de schwas maintenus ( $5 \%$ des occurrences) ratifie la prédominance des /ç/ correspondant à une réalisation en syllabe fermée. La séquence /-ml-/ reste phonologiquement prohibée (pas d'initiale de ce type, pas de formation néologique exploitant cette combinaison), en sorte qu'un schwa s'intercale dans camelote, chamelier, femmelette, grommeler, jumeler, mamelon, pommelé, ressemeler, sommelier. Pourtant, c'est la suite /-ml-/ qui surface dans $93 \%$ des cas, en comptant l'épenthèse, et même dans les cas résiduels où le vocalisme est préservé, il ne modifie pas l'aperture de la voyelle précédente. 
Sur les 12 occurrences, 8 sont imputables au témoin 78 qui est originaire du Gers dont il a conservé l'accent et qui n'amuït jamais le schwa; 1 au témoin 10 qui a passé sa petite enfance en Limousin (cf. Durand \& Tarrier 2003). On relève que d'autres locuteurs méridionaux (e.g. le témoin 45) n'ont pas conservé cette variation. Deux occurrences apparaissent dans des emphases, dans une demande de confirmation incrédule :

Exemple 1 : Témoin $21(0.34)$

$\mathrm{Q}$ : Comment est-ce qu'on fait une omelette chez vous, Madame?

$\mathrm{R}:$ /rire/ Une omelette /çməl t/?

Exemple 2 : Témoin $123(0.35)$

$\mathrm{Q}$ : Vous pouvez me décrire très précisément comment on fait une omelette ?

$\mathrm{R}$ : Une omelette /çməl $\mathrm{t} /$ ?

Dans le dernier cas, la personne (témoin 133) prononce le mot «omelette » à l'instant où un enfant, qui assistait à l'entretien, sort de la pièce en claquant la porte et la prononciation du schwa semble une façon de compenser le «bruit», dans les deux acceptions du terme. Sauf le témoin 78, tous réalisent une ou plusieurs fois le mot « omelette » sans schwa.

Quoi qu'il en soit, comme le montre l'indépendance de l'aperture du /ç/ par rapport au remplissage vocalique de la deuxième syllabe, le schwa, quand il est timbré, met en évidence la dissociation des propriétés de compte syllabique (lorsqu'il est réalisé, il accroît le mot d'une syllabe) et de coupe syllabique (sa réalisation ne rétroagit pas sur la syllabe précédente, comme le montre la conservation de l'ouverture du/ç/).

Il en résulte un contact $\mathrm{du} / \mathrm{m} /$ et $/ \mathrm{l} /$ qui aboutit à l'émergence d'un /b/ épenthétique avec ou sans nasalisation de la voyelle précédente.

Tableau 10 Epenthèse

/b/

$$
\begin{aligned}
& \text { après /Ç/ } \\
& \text { après /ç ) / }
\end{aligned}
$$

3

2

La réalisation $\mathrm{du} / \mathrm{l} /$ montre un élément plus stable puisqu'on ne constate qu'un remplacement par /w/ et deux amuïssements partiels. Là encore, comme le montrent les amorces, la présence possible d'un schwa démarcatif, la position explosive ou la présence en attaque syllabique sinon, font que ce /1/ fonctionne en réalité comme un début de mot, ou de morphème, ce qui le rend particulièrement résistant aux phénomènes de lénition.

\section{Une lecture sociologique}

On a constaté que /ç/ constituait la forme attendue et qu'elle représente exactement la moitié des occurrences, une valeur à peu près constante pour les classes A, E et I mais pour laquelle un décrochage se manifeste pour la classe $\mathrm{O}$ et qui se confirme au point de devenir minoritaire pour la classe $\mathrm{U}$.

Le choix en second de /œ/ est plus nettement représenté pour les classes A et E (un tiers des emplois), alors qu'il est moins présent dans les classes I et $\mathrm{O}$ (un peu moins de $15 \%$ des emplois). Dans la classe U, le score est intermédiaire (22\%). Les scores de I et O s'expliquent par l'importance relative des /o/ qui comptent pour 3\% des réalisations des A et $1,5 \%$ des E mais pour $20 \%$ des I et des $\mathrm{O}$ et $50 \%$ des U. Ces deux classes partagent aussi le recours au /ə/ et au /m/ (de 8 à $13 \%$ ) et toutes les classes, sauf A (qui avait 
ISBN 978-2-7598-0358-3, Paris, 2008, Institut de Linguistique Française

Sociolinguistique et écologie des langues

$$
\text { DOI } 10.1051 / \mathrm{cmlf0} 0157
$$

$2 / \mathrm{a} / \mathrm{ou} / \mathrm{m} /$ sur 30 occurrences), ont en partage des $/ \varnothing /$ et des $/$ ç $/$. . On peut donc dresser un tableau de distribution des réalisations $\mathrm{du} / \mathrm{c} /$ en assignant aux variations une valeur sociologique. 
Tableau 11 Interprétation sociologique des variations du/ç/: classes A et E

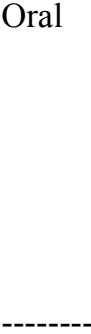

Nasal

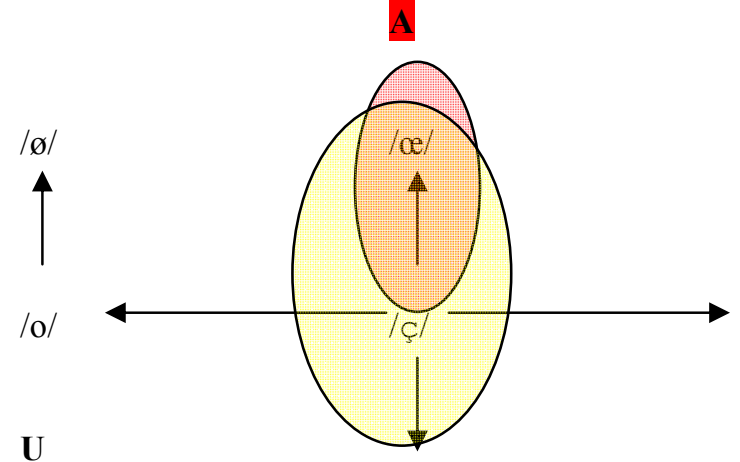

/Ç ) /
I

$/ 2 /$

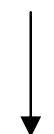

$/ m /$

fermé ouvert neutralisé

postérieur

antérieur

neutralisé

Tableau 12 Interprétation sociologique des variations du /ç/: classes I et $\mathrm{O}$

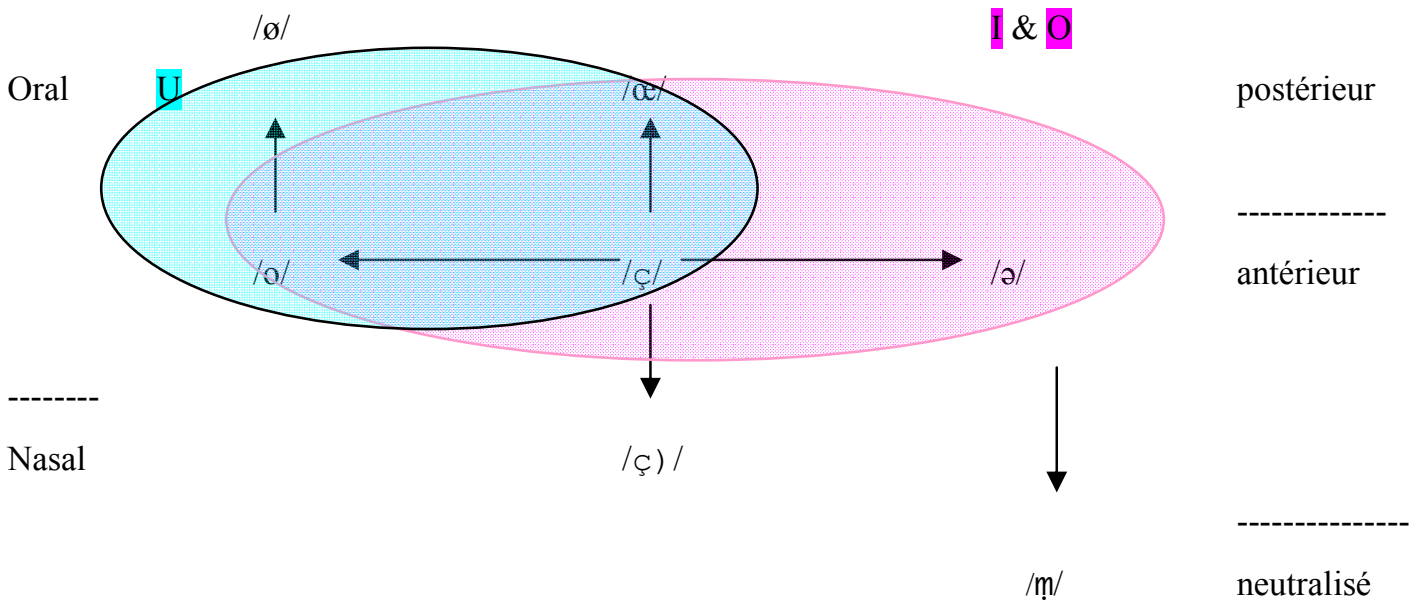

fermé ouvert neutralisé

On constate que le système de variations préserve une cible phonologique (celle qu'on suppose projetée sur le signal à des fins de comparaison et de validation) qui situe la valeur centrale non comme une entité substantielle mais comme un point d'équilibre. La variation, comme neutralisation régressive et récurrente des valeurs phonologiques, n'est pas indifférente à une caractérisation sociologique. La variation se manifeste également et différemment dans tous les groupes sociaux mais les valeurs 
neutralisées ne sont pas les mêmes en sorte que le groupe U, il est vrai restreint à 8 locuteurs et 22 occurrences, présente une distribution où /o/ constitue la moitié des formes.

\section{Conclusion}

Sur cet exemple, on a voulu montrer, à partir d'un corpus contrôlable, comment l'interdépendance de la morphologie et de la phonologie, d'une phonologie codant de l'information morphologique et d'une morphologie contraignant la phonologie, contribuait à la réalisation de variations comme effets de la morpho-phonologie. Ce n'est pas la variation qui est sociolinguistique (elle est inhérente à la structure même de la langue), c'est la préférence accordée à l'un ou l'autre des processus de neutralisation. La différence sociale s'actualise dans la distance, qu'on peut reconstruire à partir des occurrences, entre la cible phonologique et ses actualisations (Labov 1976).

Pour «omelette », la cible phonologique s'étant stabilisée, vers 1970, comme une réalisation sans vocalisation du schwa et avec /ç/, il semble qu'une double partition se dessine (des dialectes, donc) :

- un dialecte diatopique, opposant prononciation méridionale et prononciations standard (cf. Durand),

- un dialecte diastratique opposant à tous les autres groupes la catégorie la plus dominée qui apparait conservatrice dans sa réalisation, comme si elle préservait la syllabation induite par la réalisation du schwa.

C'est ce que confirmerait l'étude de la suite des phonèmes d'omelette. C'est ce qu'on devra vérifier dans ESLO2.

Le corpus « Omelettes / ESLO1 / LLL » est disponible sur le site de la DGLFLF.

\section{Bibliographie}

Bernstein, B. (1975) Langage et classes sociales, Minuit.

Bourdieu, P. (1979) La Distinction, Minuit.

Durand J., Laks B. \& Lyche Ch. eds (2003) «La prononciation du français dans sa variation », La Tribune internationale des langues vivantes 33.

Durand J. \& Tarrier J.-M. (2003) «Enquête phonologique en Languedoc» in Durand J. \& al., 2003 : 117 127.

Edmont, E \& Gilliéron, J. (1902-1910) L'Atlas linguistique de la France, Champion.

Encrevé, P. (1988) La liaison avec et sans enchaînement: phonologie tridimensionnelle et usage du français, Paris, Seuil.

Gadet, F. (1989) Le français ordinaire, A. Colin.

Labov, W. (1976) Sociolinguistique, Minuit.

Laks, B. (1977) «Contribution empirique à l'analyse socio-différentielle de la chute de /r/ dans les groupes consonantiques finals, Langue française $34: 109-125$.

Le Berre, Y., Le Dû J. \& Brun-Trigaud G. (2004) Lectures de l'Atlas Linguistique de la France de Gilliéron et Edmont, CTHS.

Scheer, T. (2004) A Lateral Theory of Phonology. What is CVCV, and why should it be ? Berlin-New York, Mouton de Gruyter.

Ségéral, Ph. \& Scheer, T. (2001) « La Coda-Miroir », Bulletin de la Société de Linguistique de Paris 96, $1: 107-152$. 